\title{
Assistive environments for the disabled and the senior citizens: theme issue of PETRA 2010 and 2011 conferences
}

\author{
Ilias Maglogiannis • Margrit Betke • \\ Grammati Pantziou • Fillia Makedon
}

Published online: 4 November 2012

(c) Springer-Verlag London 2012

The introduction of pervasive and ubiquitous systems into everyday life pushes this technology to become increasingly embedded in artifacts and environments trying to address an important social and health care issue: as the world's population ages, there is growing need for technologies to assist with the in-home care of the elderly and people with Alzheimer's, Parkinson's and numerous types of chronic disabilities or traumas. The quality of people's living environments is particularly important in an increasingly crowded and complex world in which inclusiveness and connectivity with the rest of the world is critical. Furthermore, enabling such persons remain productive and active is of high socioeconomic importance to every country's workforce or health care system. Assistive environments is a field that addresses the fact that, as people grow older or become disabled, they increasingly rely on computer technology to be able to live in and function in their homes [1-3]. Finally, assistive environments is a field that pushes innovation and interdisciplinary collaboration forward, catalyzes the union of computation

I. Maglogiannis ( $\square)$

Department of Computer Science and Biomedical Informatics, University of Central Greece, Papasiopoulou 2-4,

35100 Lamia, Greece

e-mail: imaglo@gmail.com; imaglo@ucg.gr

\section{Betke}

Department of Computer Science, Boston University, Boston, MA, USA

\section{G. Pantziou}

Department of Informatics, Technological Educational Institution of Athens, Athens, Greece

F. Makedon

Heracleia Computing Lab, University of Texas at Arlington, Arlington, TX, USA with the development of new materials (e.g., sensors) and devices (e.g., assistive robots), new or improved drugs and drug administration, new methods and software for rehabilitation, physical/occupational therapy, new materials for safety or disorder monitoring (e.g., sleep disorders connected with epilepsy or depression), new algorithms for mental health and emotion monitoring (e.g., through facial expressions or speech), new types of virtual life coaches (e.g., avatars to promote exergaming) and many other innovations.

This special issue comes after the successful organization of the $3 r d$ and 4th International Conferences on "PErvasive Technologies Related to Assistive Environments-PETRA 2010 and 2011," which were held in Corfu, Greece and Herakleion, Crete, Greece, respectively (http://www.petrae.org/). Both conferences received funding from the US National Science Foundation as well as support from the Hellenic National Center for Scientific Research-Demokritos and many other organizations.

The focus of this special issue is how and what new technological innovations can assist and empower an elder or disabled human in everyday life. What technologies can assist specific health care needs, at home and at work, in a person's everyday routine by using sensors, databases, personal area networks, ubiquitous connectivity, distributed intelligence and adaptive, easy to use interfaces and interactions. This special issue focuses on research involving the design, development, evaluation and use of emerging pervasive technologies for assistive environments for the disabled and the senior citizen, taking also into account the target audience of the PUC journal.

The realization of pervasive health information applications through mobile devices introduces several challenges, such as (1) infrastructure for unified and ubiquitous access or medical data storage and management [4, 5], 
(2) appropriate Human Computer Interfaces (HCI) especially designed for the disabled and the senior citizen, (3) security and privacy combined with location services [6] and (4) context awareness and intelligent modules able to automatically process the data provided by medical devices and sensors, exchange knowledge and make intelligent decisions in a given context [7, 8]. These are the aspects that we try to cover in this special issue.

For example, in the area of intelligent and context-aware systems, Jara et al. [9] present a pervasive Pharmaceutical Intelligent Information System (PIIS) based on the Internet of Things (IoT) for drug identification and monitoring of medication. The authors demonstrate how the IoT is applied to examine drugs in order to fulfill treatment, to detect harmful side effects of pharmaceutical excipients, allergies, liver/renal contradictions and harmful side effects during pregnancy, in order to improve quality assurance in drug delivery, to improve adherence to drug appropriate consumption, and to reduce clinical errors caused by dosage mistakes and drug interactions.

Metsis et al. [10] deal in their paper with the monitoring of sleep patterns. This topic is quite important for various reasons such as the detection and treatment of sleep disorders, the assessment of the effect of different medical conditions or medications on the sleep quality and the assessment of mortality risks associated with sleeping patterns in adults and children. Sleep monitoring by itself is a difficult problem due to both privacy and technical considerations. The authors present in their paper a system that uses a combination of noninvasive sensors to assess and report sleep patterns: a contactbased pressure mattress and a non-contact 3-D image acquisition device, which can complement each other. The system is evaluated on real data collected in Heracleia Lab's assistive living apartment.

Wang et al. [11] present a learning algorithm for automatically classifying patient thought records (TR). TR categorization is important in cognitive behavior therapy (CBT), which is a useful augmentation of standard clinic treatment for major depressive disorder (MDD). The authors propose a general framework to represent the TR text in a new domain based on self-learning algorithm proposed from the machine learning literature. With the help of introducing a large number of unlabeled dataset, the self-learning algorithm is expected to find a better feature representation, which is cast as a sparse coding problem.

In the HCI domain, Caridakis et al. [12] present in their work the incorporation of non-manual cues in automatic sign language recognition. More specifically, eye gaze, head pose and facial expressions are discussed in relation to their grammatical and syntactic function, and means of including them in the recognition phase are investigated. Computer vision issues related to extracting facial features, eye gaze and head pose cues are presented, and classification approaches for incorporating these non-manual cues into the overall sign language recognition architecture are also introduced, and the corresponding results are presented.

The work by Epstein et al. [13] also falls within the HCI domain for disabled persons and especially for people who cannot use their hands to control a computer mouse due to conditions such as cerebral palsy or multiple sclerosis. For these individuals, Epstein et al. propose to significantly improve a popular mouse-replacement solution called Camera Mouse that converts head motions captured with a web camera into mouse pointer coordinates on the screen. The Camera Mouse uses an optical flow approach to track a small patch of the subject's face, such as the nostril or the edge of the eyebrow. It may lose the facial feature when the tracked image patch drifts away from the initially selected feature or when a user makes a rapid head movement. To address the problem of feature loss, the authors have developed and incorporated the Kernel-Subset-Tracker into the Camera Mouse. The Kernel-Subset-Tracker is an exemplar-based method that uses a training set of representative images to produce online templates for positional tracking. The conducted experiments with test subjects demonstrate that augmenting the Camera Mouse with the Kernel-Subset-Tracker improves communication bandwidth statistically significantly.

Belsis and Pantziou [14] deal with the important issue of citizen's privacy protection in assistive environments. In particular, they present a privacy-preserving architecture, which builds upon the concept of k-anonymity. A clustering-based anonymity scheme for effective network management and data aggregation, which also protects user's privacy by making an entity indistinguishable from other $\mathrm{k}$ similar entities, is proposed. The presented algorithm is "resource aware," as it minimizes energy consumption with respect to other more costly, cryptography-based approaches. The proposed system is evaluated from an energy consuming and network performance perspective, under different simulation scenarios.

Drosatos and Efraimidis [15] apply a version of the Nearest Doctor Problem (NDP) for finding the nearest doctor in case of an emergency and present a privacypreserving protocol for solving it. The presented solution is based on cryptographic primitives and makes use of the current location of each participating doctor. The implemented protocol tries to protect the privacy of the doctors' locations as well. A prototype for a community of doctors that use mobile devices to obtain their current location is presented and evaluated in the paper.

The last two papers of the special issue fall in the domain of assistive environment infrastructures. Poulymenopoulou et al. [16] develop mechanisms to deal with emergency care management based on the assessment of individual needs. The authors present a coordinated plan of 
care that is consistent with agreed priorities and is designed to bring optimal outcomes using cost-effective care. On these grounds, they present the development of a workflow-based electronic patient record (E-EPR) for emergency care, which is provided as a service on a cloud. The E-EPR aims at supporting the execution of emergency workflows by providing integrated citizen information to emergency process participants and to make this information shareable to health and social care professionals. Due to the heterogeneity of the participating organizations' information systems, the E-EPR is built on top of an Integrating the Healthcare Enterprise (IHE)-based collaborative network consisted of emergency agencies and health and social care providers that share patient documents using IHE-based profiles. An experimental implementation of the E-EPR is also described in their paper.

In the last paper, Anagnostopoulos et al. [17] show optimizations for Wireless Sensor Networks (WSNs) used in assistive applications and show how these are influenced by many factors such as transmission errors and power consumption. The authors propose a multivariate context-forwarding model that achieves energy efficient WSN operation. A networking node adopts multivariate auto-regression for forecasting contextual information (biosignals or vital parameters) and locally decides whether context retransmission is required or not. This scheme is applied in patient telemonitoring systems where accurate yet energy-aware transmission of biosignals to a remote control unit is crucial. Simulation results are reported indicating the capability of the proposed model in minimizing energy consumption in WSNs having as application domain the pervasive assistive applications.

As guest editors, in this special issue, we tried to cover a broad area of topics by selecting papers that are representative of assistive environments research and thus give the reader an overview of the immense potential of the field. From our position, we wish to thank all the authors for their contributions and the reviewers for assisting our editorial work. We do hope that the papers included in this special issue will satisfy the audience of the PUC Journal and readers will find them interesting.

\section{References}

1. Maglogiannis I, Makedon F, Pantziou G, Baillie L (2010) Pervasive technologies for assistive environments: special issue of PETRA 2008 conference. Pers Ubiquit Comput 14(6):469-471
2. Doukas C, Metsis V, Becker E, Le Z, Makedon F, Maglogiannis I (2011) Digital cities of the future: extending @ home assistive technologies for the elderly and the disabled. Telematics Inform 28(3): 176-190

3. Doukas C, Maglogiannis I (2011) Emergency fall incidents detection in assisted living environments utilizing motion, sound and visual perceptual components. IEEE Trans Inf Technol Biomed 15(2):277-289

4. Doukas C, Maglogiannis I (2008) Adaptive transmission of medical image and video using scalable coding and context-aware wireless medical networks. EURASIP J Wirel Commun Netw Hindawi Press, vol 2008, p 12, Article ID 428397. doi:10.1155/2008/428397

5. Antoniadou E, Maglogiannis I (2010) Adoption of wearable systems in modern patient telemonitoring systems. In: CruzCunha MM, Tavares AJ, Simoes R (eds) Handbook of research on developments in e-Health and telemedicine: technological and social perspectives. IGI Global, Hershey, pp 1004-1023

6. Maglogiannis I, Kazatzopoulos L, Delakouridis K, Hadjiefthymiades S (2009) Enabling location privacy and medical data encryption in patient telemonitoring systems. IEEE Trans Inf Technol Biomed 13(6):946-954

7. Maglogiannis I (2009) Introducing intelligence in electronic healthcare systems: state of the art and future trends. Artif Intell Int Perspect LNAI 5640:71-90

8. Doukas C, Maglogiannis I (2010) Advanced classification and rules-based evaluation of motion, visual and biosignal data for patient fall incident detection. Int $\mathbf{J}$ Artif Intell Tools (IJAIT) special issue on "Artificial Intelligence Techniques for Pervasive Computing" 19(2):175-191

9. Jara AJ, Zamora MA, Skarmeta AF (2012) Drug identification and interaction checker based on IoT to minimize adverse drug reactions and improve drug compliance. Pers Ubiquit Comput. doi:10.1007/s00779-012-0622-2

10. Metsis V, Kosmopoulos D, Athitsos V, Makedon F (2012) Non-invasive analysis of sleep patterns via multimodal sensor input. Pers Ubiquit Comput. doi:10.1007/s00779-012-0623-1

11. Wang H, Huang H, Basco M, Lopez M, Makedon F (2012) Selftaught learning via exponential family sparse coding for costeffective patient thought record categorization. Pers Ubiquit Comput. doi:10.1007/s00779-012-0614-2

12. Caridakis G, Asteriadis S, Karpouzis K (2012) Non-manual cues in automatic sign language recognition. Pers Ubiquit Comput. doi:10.1007/s00779-012-0615-1

13. Epstein S, Missimer E, Betke M (2012) Using kernels for a videobased mouse-replacement interface. Pers Ubiquit Comput. doi: 10.1007/s00779-012-0617-z

14. Belsis P, Pantziou G (2012) A $k$-anonymity privacy-preserving approach in wireless medical monitoring environments. Pers Ubiquit Comput. doi:10.1007/s00779-012-0618-y

15. Drosatos G, Efraimidis PS (2012) An efficient privacy-preserving solution for finding the nearest doctor. Pers Ubiquit Comput. doi: 10.1007/s00779-012-0619-x

16. Poulymenopoulou M, Malamateniou F, Vassilacopoulos G (2012) E-EPR: a workflow-based electronic emergency patient record to support holistic care management. Pers Ubiquit Comput. doi: 10.1007/s00779-012-0620-4

17. Anagnostopoulos C, Hadjiefthymiades S, Katsikis A, Maglogiannis I (2012) Autoregressive energy-efficient context forwarding in wireless sensor networks for pervasive healthcare systems. Pers Ubiquit Comput. doi:10.1007/s00779-012-0621-3 